\title{
Clustering of Dust Particles on Water Surface
}

\author{
M. Rapini, A.S. Chaves, and B.V. Costa \\ Departamento de Física, ICEX - Universidade Federal de Minas Gerais \\ Caixa Postal 702, 30123-970, Belo Horizonte, MG, Brazil
}

Received on 8 August, 2004

\begin{abstract}
In this work we use molecular dynamics simulation to study the clustering of dust particles deposited on a liquid surface. Our results are compatible with the picture that one particle attracts another due to the deformation of the liquid medium which creates an attractive potential around the deposited particle. The similarity to other physical phenomena is discussed.
\end{abstract}

\section{Introduction}

In our daily life we observe many physical phenomena. Some are so common that our senses tell us to ignore them. However, that is only a reflex behavior, just trying to keep our attention on a more important job. It is not uncommon that observing a simple phenomenon we are not able to respond a question like: why is that so? In many cases we even haven't thought about it. We intend in this work to discuss one such phenomenon. If we carefully observe the surface of water in a glass which we let sit in our yard for a day, we will notice that small dust particles, deposited on the surface, are agglomerated in large clusters as in Fig. 1.

This curious effect may have several explanations. For example

- The dust particles may be electrically charged with opposite charges, being attracted to each other;

- The movement of the water create regions of maximum and minimum on the surface, the dust particles may accumulate in some of those minima;

- Particles deposited on the water surface may deform the region around them creating a minimum (See Fig. 2). When another particle falls close to the first one, their minima interact in such a way as to create an attractive potential between them.
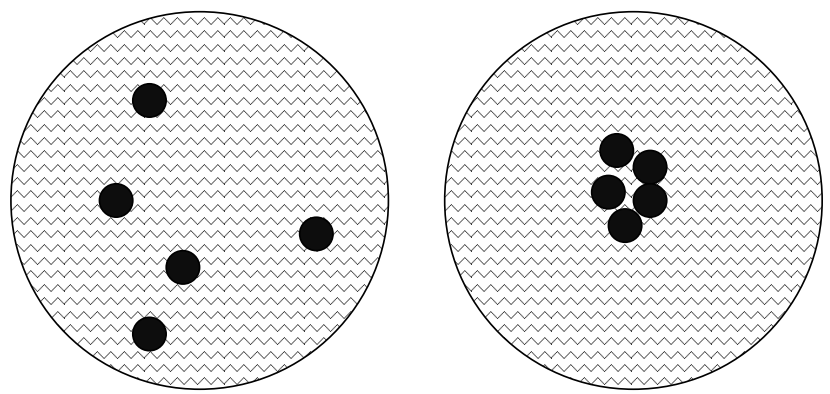

Figure 1. Small dust particles, deposited on the surface of water agglomerate into large clusters. Why?

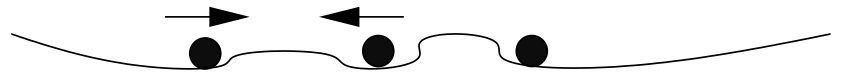

Figure 2. Particles deposited on the water surface deform the surrounding region, creating an attractive potential for other particles.

In this work we use molecular dynamics simulation to study the phenomenon described above. We simulate the water surface as a membrane formed by an array of particles of mass $m$ connected by springs of elastic constant $k$ (See Fig. 3).

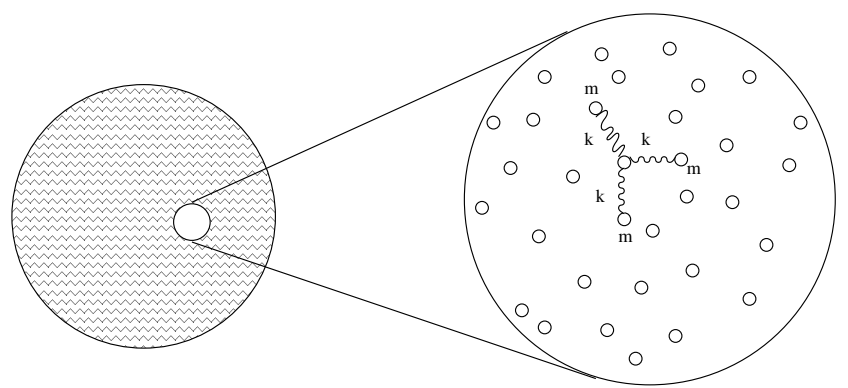

Figure 3. Membrane formed by an array of particles of mass $m$ connected by springs of elastic constant $k$ simulating the water surface.

Particles are labelled from 1 to $N$. The system dimensions are $L_{x}$ and $L_{y}$. The potential between particles is taken as harmonic

$$
V_{\text {spring }}\left(r_{i}, r_{j}\right)=k\left[\left(r_{i}-r_{j}\right)-\left(r_{0 i}-r_{0 j}\right)\right],
$$

where $r_{0 n}$ is the equilibrium position of particle $n$. The particles deposited on the membrane interact with particles on the surface and between themselves through a LennardJones potential,

$$
V_{L J}\left(r_{\mu}, r_{\nu}\right)=4 \epsilon\left[\left(\frac{\sigma}{r_{\mu, \nu}}\right)^{12}-\left(\frac{\sigma}{r_{\mu, \nu}}\right)^{6}\right]
$$



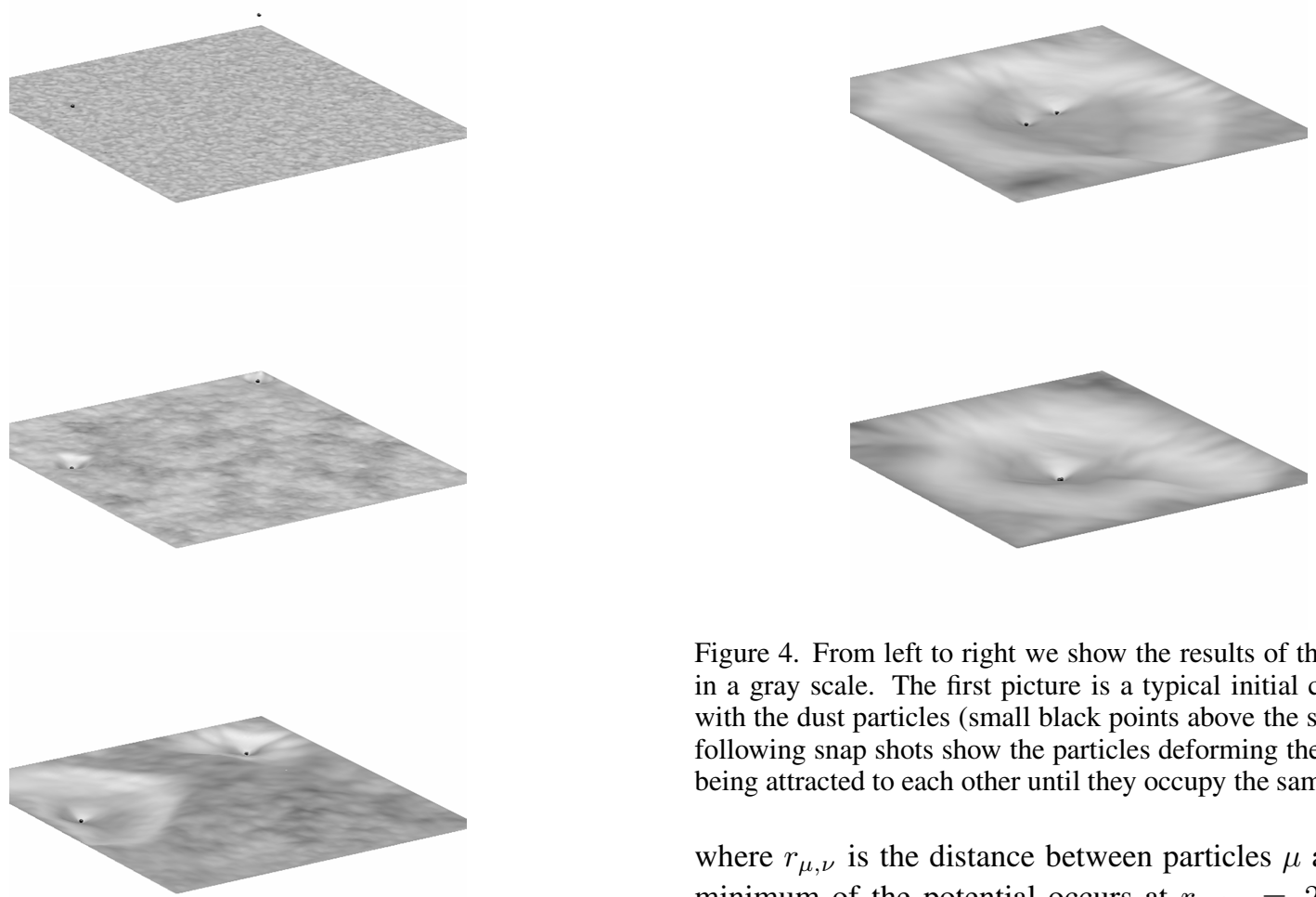

From left to right we show the results of the simulation in a gray scale. The first picture is a typical initial configuration with the dust particles (small black points above the surface). The following snap shots show the particles deforming the surface and being attracted to each other until they occupy the same minimum.

where $r_{\mu, \nu}$ is the distance between particles $\mu$ and $\nu$. The minimum of the potential occurs at $r_{\min }=2^{1 / 6} \sigma$. The parameters $\epsilon$ and $\sigma$ are chosen to fit the condition that the attractive term is very small in comparison to the system size. With this choice we get a hard sphere interaction between dust particles. The equation of motion for each particle is obtained by writing

$$
F_{n}=-\vec{\nabla}_{n}\left[\sum_{j} V_{\text {spring }}\left(r_{n}, r_{j}\right)+\sum_{\nu} V_{L J}\left(r_{n}, r_{\nu}\right)\right] .
$$

The equation of motion were integrated forward in time by using a fourth order Runge-Kutta method[1]. Our results, shown in Fig. 4, agree with the hypothesis that a particle deposited on the surface modifies the medium creating an attractive potential around it. There are similar effects observed in several physical phenomena, for example the deformation of space in presence of a mass[2] or the formation of cooper pairs, in superconductivity, due to the presence of a phonon[3].

\section{Acknowledgements}

Work partially supported by $\mathrm{CNPq}$ and FAPEMIG

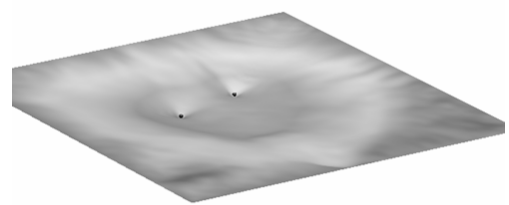
(Brazilian agencies) and CIAM-02 49.0101/03-8 (CNPq). Numerical work was done in the LINUX parallel cluster at the Laboratório de Simulação Departamento de Física UFMG.

\section{References}

[1] Computational Physics, J.M. Thijssen, Cambridge University Press (1999).

[2] Gravitation and Cosmology : Principles and Applications of the General Theory of Relativity, Steven Weinberg, (1999)

[3] Quantum Physics of Atoms, Molecules, Solids, Nuclei, and Particles, Robert Eisberg and Robert Resnick, John Wiley and Sons (1974). 